ISSN electrónico: 2445-1355

DOI: http://dx.doi.org/10.14201/fj2020516573

\title{
EFECTO DE LA EXPOSICIÓN A OZONO SOBRE UN MODELO DE PRIVACIÓN MATERNA EN RATAS
}

\section{Effect of ozone exposure on a rat model of maternal deprivation}

Carmen MOYA; Joaquín GONZÁLEZ

Facultad de Farmacia, Universidad de Castilla-La Mancha (UCLM). Avda. Doctor José María Sánchez Ibáñez s/n. 02008 Albacete. Tfno. 967599 200. Ext: 22.36

Correo-e: joaquin.gfuentes@uclm.es

RESUMEN: El maltrato infantil es uno de los mayores problemas sociales y económicos a nivel mundial. Entre las personas que han sido víctimas del MI existe una alta prevalencia de violencia, abuso de drogas y enfermedades psiquiátricas, entre otros. A nivel fisiológico, el eje hipotálamo-pituitariaadrenal y la respuesta al estrés están directamente relacionados con esta prevalencia. Además, en los últimos años, ha aumentado la preocupación por el efecto de la contaminación sobre la salud humana. El objetivo de este proyecto es determinar el efecto de la exposición crónica a un ambiente contaminado con ozono, sobre algunos biomarcadores, en un modelo animal de privación materna. Se medirá cómo afecta la exposición a ozono sobre la capacidad cognitiva y el estado de ansiedad. Además, se analizará la variación de corticosterona y ACTH en plasma tras la exposición a un estresor agudo. Por último, se analizará el volumen del hipocampo y sus subáreas, así como el grado de metilación del exón promotor del receptor de glucocorticoides $1 \mathrm{~F}$ y del exón promotor de vasopresina. Con el presente trabajo se pretende ampliar el conocimiento sobre el desarrollo cognitivo de niños que han sufrido maltrato infantil.

Palabras clave: Maltrato infantil; ozono; desarrollo cognitivo.

ABSTRACT: Childhood maltreatment is one of the biggest social and economic problems worldwide. Among people affected by Childhood

Ediciones Universidad de Salamanca / @@ఠ $\quad$ FarmaJournal, vol. 5, núm. 1 (2020), pp. 65-73 
maltreatment there is a high prevalence, inter alia, of violence, drugs abuse and psychiatric disorders. At the physiological level, the HypothalamicPituitary-Adrenal axis and the stress response are key to this prevalence. Moreover, in recent years, pollution has become an important issue because it affects directly human health. The objective of this experiment is to elucidate the effect of a chronic exposure to pollution, in particular ozone, on some biomarkers in a maternal deprivation animal model. We will measure how ozone exposure affects cognitive ability as well as anxiety state. Besides, the plasma variation of corticosterone and ACTH after an acute stressor will be analysed. We will also see if hippocampus and hippocampal subareas volume is altered in the animal model after the exposure to ozone. Finally, we will evaluate the methylation state of the exon promotor of glucocorticoid - receptor $1 \mathrm{~F}$ and the exon promotor of vasopressin. This study is thought to start looking for the answer to how pollution affects the cognitive development of children who have suffered from Childhood maltreatment.

Key words: Child abuse; ozone; cognitive development.

\section{INTRODUCCIÓN}

Existen periodos durante los cuales el cerebro es especialmente sensible a los estímulos del entorno. Uno de estos periodos comprende desde la etapa prenatal hasta los 2 años (Dobbing, Sands, 1973). Durante esta etapa, distintas experiencias reforzarán unas conexiones y eliminarán otras, produciendo cambios, que pueden mantenerse hasta la edad adulta (Andersen, Teicher, 2004; Buss et al., 2012).

Muchos de los factores inmunológicos y endocrinos importantes para el desarrollo y el crecimiento neurológico también están involucrados a nivel central en la respuesta al estrés (Buss et al., 2012) y, por tanto, el estrés juega un papel determinante en el desarrollo del cerebro.

El ser humano vive en un entorno en constante cambio. El estrés, y la respuesta fisiológica que conlleva, es una de las formas de adaptación a esos cambios. A rasgos generales, mientras que el estrés agudo es una pieza clave para la adaptación, la cronicidad del estrés produce una disrupción continuada de la homeostasis, pudiendo desarrollarse efectos negativos a largo plazo. Además, que el estrés nos afecte positiva o negativamente depende de factores como el entorno social, la genética y el tipo de estresor. Algunos de estos factores están determinados, en ocasiones, por decisiones personales, sin embargo, otros se escapan de nuestro control. Por ejemplo, mientras que sufrir un evento estresante durante la niñez 
(Early life Stress, ELS) es un hecho que no podemos controlar, el lugar donde vivimos y, por tanto, el tipo de aire y estresores que respiramos, pueden estar condicionados por nuestras decisiones (Sapolsky 2015).

\subsection{Respuesta al estrés}

Superar el estrés de forma efectiva implica que la respuesta a éste se active de forma rápida y finalice cuando el estresor deje de estar presente. En este proceso están involucrados el sistema autónomo, que se activa de forma inmediata produciendo la liberación de noradrenalina (NA) y adrenalina, y el sistema endocrino que, mediante el eje hipotálamo-pituitaria-adrenal (HPA) (Fig 1), produce una concentración máxima de corticoides (Co) en 15-20 minutos (Buss et al., 2012).

Figura 1. Eje Hipotálamo-Pituitaria-Adrenal

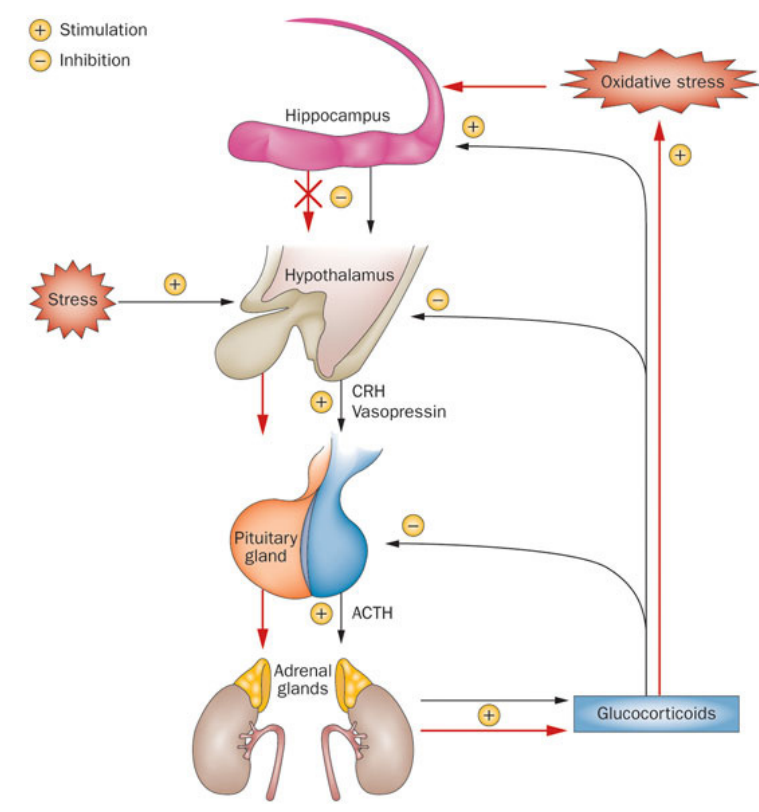

Una disrupción de la homeostasis produce la liberación de la hormona liberadora de corticotropina (CRH) y de la vasopresina (AVP) desde el núcleo paraventricular (PVN). Estas estimulan la liberación de la hormona adrenocorticotropa (ACTH) desde la pituitaria anterior, la cual actúa sobre las cortezas adrenérgicas liberando glucocorticoides (Miller, O’Callaghan, 2002). Los glucocorticoides

Ediciones Universidad de Salamanca / @@ $\quad$ FarmaJournal, vol. 5, núm. 1 (2020), pp. 65-73 
se distribuyen por el cuerpo y proporcionan un feedback negativo al sistema a través de la pituitaria, el hipotálamo y el hipocampo (Hip) (Kaufman et al., 2000), mediante la activación de los receptores glucocorticoides (GCr) (Buss et al., 2012).

\subsection{Maltrato en la infancia}

El maltrato infantil (MI) es un problema a nivel mundial, con una prevalencia del 15\% (Gilbert et al., 2009). Según UNICEF 6/10 niños en el mundo de entre 2 y 14 años sufren maltrato físico a diario (Europapress, 2014). En las últimas décadas numerosos estudios epidemiológicos han apoyado la hipótesis de que sufrir un evento estresante, durante la niñez (early life stress, ELS), aumenta el riesgo de padecer déficits cognitivos, depresión, drogodependencia, comportamientos suicidas y violentos, así como peor respuesta a los tratamientos (Nemeroff, 2016). El Centro de Control y Prevención de Enfermedades estima que la erradicación del MI reduciría $1 / 2$ la tasa de depresión, 2/3 la de alcoholismo y 3/4 la de suicidio, abuso de drogas y violencia doméstica (van der Kolk, 2016).

En los últimos años, se ha visto que los procesos epigenéticos parecen ser determinantes para las consecuencias a largo plazo del ELS (Hertzman, 2012). Pacientes suicidas que habían sido víctimas del MI tenían disminuida la expresión del GCr en el Hip, por un aumento en la metilación del exón promotor del GCr 1F (Lutz, Turecki, 2014).

En modelos animales se ha observado que ratas que han recibido cuidados adecuados durante la infancia tienen aumentada la expresión del GCr (Kaufman et al., 2000), la longitud dendrítica y densidad sináptica en el Hip y disminuido el ARNm de CRH en el PVN. También observaron que, ante distintos estresores, estas ratas muestran menor nivel de NA en el PVN (Nemeroff, 2016) y menor aumento de ACTH y corticosterona, con un rápido descenso a niveles basales (Kaufman et al., 2000). Por otro lado, a nivel conductual, cuanta más atención recibían las crías, menor ansiedad presentaban (Miller et al., 2016).

Además de este modelo animal, existen otros en los que el ELS se simula separando a las crías de sus madres durante los primeros días de vida (privación materna, PM). Estas crías mostraron un aumento de la expresión de AVP, de la secreción de corticosteroides y de la activación de PVN. Además, mostraron mayor comportamiento de desesperación y una disminución de la memoria. Si este modelo se prolongaba a 6 semanas, se empezaban a ver cambios epigenéticos como la hipometilación del promotor de AVP (Lutz, Turecki, 2014).

Ediciones Universidad de Salamanca / @@ఠ $\quad$ FarmaJournal, vol. 5, núm. 1 (2020), pp. 65-73 


\subsection{Contaminación atmosférica}

Según la OMS, el 92\% de la población mundial vive en lugares donde el nivel de calidad del aire excede los límites fijados, provocando unas 3 millones de muertes al año (WHO 2016).

En las últimas décadas, se ha señalado que el aire contaminado afecta, además de a las vías respiratorias, a otros órganos, y que está relacionado con enfermedades crónicas como hipertensión o enfermedades neurodegenerativas. En los últimos años el interés sobre el ozono ha aumentado no sólo por el efecto que tiene sobre la salud, sino, también, porque se espera que su concentración aumente como consecuencia del cambio climático (Kodavanti, 2016).

Un estudio reciente confirmó que los efectos perjudiciales causados por el ozono son debidos a la activación de la respuesta simpática y endocrina al estrés (Kodavanti, 2016). Aunque los estudios en humanos son limitados, se ha demostrado que la exposición aguda a ozono produce un aumento de la concentración de cortisol. En ratas, la exposición al ozono disminuye la densidad de botones sinápticos en el Hip, aumenta los niveles de corticosterona y ACTH, y aumenta la activación del tronco cerebral, del hipotálamo, Amy y LC. Además, disminuye la capacidad cognitiva (Miller et al., 2016).

Puesto que el ELS y el ozono producen cambios fisiológicos mediante la activación del eje HPA, se hace necesario un estudio que comience a dilucidar de qué forma afecta la contaminación al desarrollo cognitivo y conductual en infantes que han sufrido algún tipo de MI. En este estudio se pretende determinar si la intervención terapéutica realizada sobre un niño que ha sufrido MI puede verse condicionada por el lugar de residencia.

\section{Objetivos}

Se plantea la hipótesis de que la exposición a ozono podría afectar de forma negativa al desarrollo de animales que han sufrido PM. El objetivo que se propone en este estudio es determinar de qué forma afecta el ozono a los siguientes parámetros:

- Capacidad cognitiva: análisis de la memoria espacial.

- Estado de ansiedad: ponderación entre la motivación de explorar y la aversión que experimentan los animales en un laberinto.

- Respuesta al estrés: cuantificación de los niveles en sangre de corticosterona y ACTH tras la exposición aguda a un estresor.

- Epigenética: determinar el grado de metilación del exón promotor de GCr 1F y del exón promotor de AVP. 


\section{Materiales y métodos}

\subsection{Procedimiento experimental}

Se utilizarán 17 parejas de ratas Wistar, que se mantendrán bajo condiciones estándar de animalario. El agua y la comida estarán disponibles ad libitum. Todos los procedimientos tendrán que ser aprobados por el Comité de Ética de Experimentación Animal.

Se espera que nazcan entre 5 y 10 crías de cada pareja. En el tercer día postnatal (P3), las crías se dividirán en dos grupos $(\mathrm{n}=40)$ : uno será sometido a PM, el otro no lo será. Tras la PM, cada grupo se dividirá en dos subgrupos $(\mathrm{n}=20)$ : uno se expondrá durante 5 meses a altas concentraciones de ozono, el otro se mantendrá en un ambiente normal.

FIgura 2. Procedimiento experimental. PM: Privación materna;

Lab. B: Laberinto Barnes; Lab. C: Laberinto en cruz

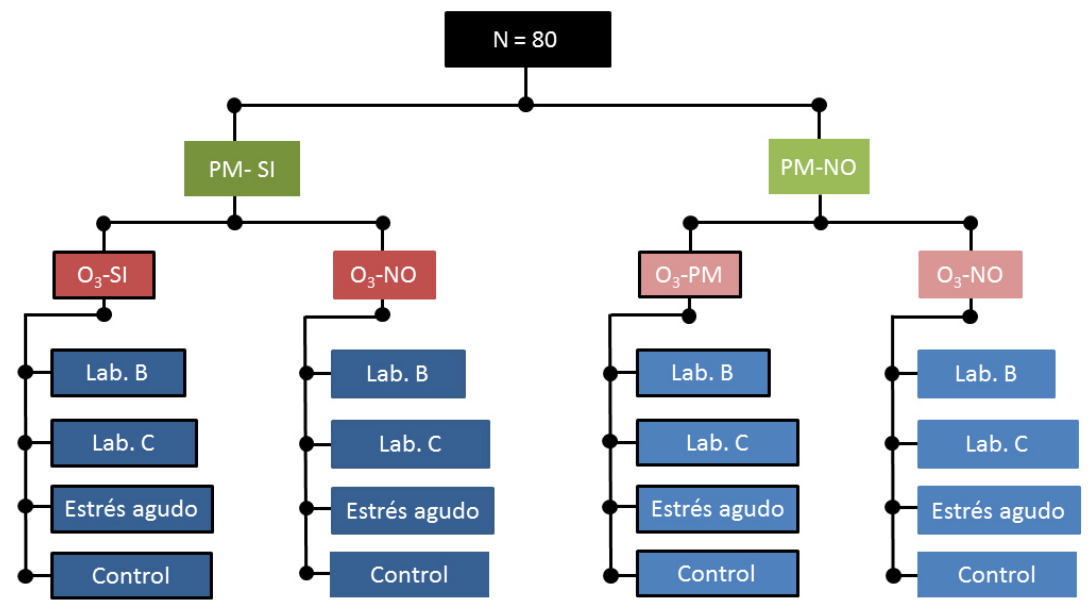

Transcurridos estos 5 meses, cada grupo de 20 animales será dividido en 4 grupos $(\mathrm{n}=5)$.

Para someter a los animales a PM las crías serán separadas de sus madres y puestas en cajas individuales 3 horas al día desde P3 al destete. Las crías pertenecientes a los grupos control se mantendrán en la caja inicial con sus madres.

La exposición a ozono se realizará mediante un sistema de ventilación individual (BeiJing Biolaunching Technologies Co., Ltd.,). Los animales estarán expuestos a una concentración de 0.25 ppm $\pm 10 \%$ durante 5 meses. 


\subsection{Análisis del comportamiento}

\section{- Capacidad cognitiva}

El laberinto de Barnes consiste en una plataforma circular con huecos circulares situados en el perímetro de la plataforma. Todos los huecos tienen un falso fondo excepto uno, debajo del cual se coloca una caja de escape. La medida de la memoria espacial se basa en la habilidad del sujeto para encontrar el hueco de escape.

Cada rata será sometida a 2 entrenamientos por día, durante 7 días, con una separación mínima de 30 minutos. Después, se realizará el test 12 horas (memoria a corto plazo) y 48 horas después del último ensayo (memoria a largo plazo).

Se medirán: tiempo de latencia, tasa de error, velocidad, tiempo en movimiento, y estrategia de búsqueda.

\section{- Estado de ansiedad}

Para valorar este punto se usará el laberinto elevado en cruz. Consiste en 4 brazos en forma de cruz unidos por una plataforma central. Dos de los brazos están abiertos al exterior (BA) y dos están cerrados (BC).

El test consiste en colocar a la rata en la plataforma central y permitirle la exploración del laberinto durante 5 minutos, para evaluar el estado de ansiedad de las ratas. Se medirán: tiempo de latencia, entradas totales a los brazos, entradas a BA, entradas a BC, tiempo en cada sección y comportamiento de no-exploración.

\subsection{Respuesta al estrés}

Las ratas serán sometidas a estrés agudo mediante la inmersión durante 15 minutos en un tanque de agua fría $\left(15^{\circ} \mathrm{C}\right)$. Se tomará 1 muestra de sangre de 400 $\mu \mathrm{L}$ antes del test y $3(1 / 30 \mathrm{~min})$ después de este.

Los niveles de corticosterona y ACTH se analizarán mediante kits comerciales (Cat No: ADI-901-097; Enzo Life Sciences. Cat No: M046006; MD BioSciences).

\subsection{Epigenética}

Los animales serán sacrificados por decapitación. Posteriormente se disecarán el hipocampo y el hipotálamo. Para la extracción del ADN de las muestras se usará el kit DNeasy (Quiagen, DNeasy Blood \& Tissue Kits). Para analizar el estado de metilación del exón promotor del GCr 1F y de AVP se hará un mapeo por bisulfito seguido de una amplificación por PCR.

Ediciones Universidad de Salamanca / @@ఠ $\quad$ FarmaJournal, vol. 5, núm. 1 (2020), pp. 65-73 


\subsection{Análisis estadístico}

Para elegir el test adecuado se analizará la normalidad de los datos, así como la homogeneidad de varianzas. Además, se tendrá en cuenta si los datos proceden de muestras pareadas o muestras independientes. Se considerarán diferencias significativas si $\mathrm{p}<0,05$.

\section{Posibles Resultados y disCUSIÓN}

Las personas que han sufrido MI tienden a ser más agresivas, tienen mayor riesgo de sufrir enfermedades y responden peor a los tratamientos. La contaminación es otro factor que puede aumentar dicha probabilidad y, junto con el maltrato, produce la sobreactivación del eje HPA (Nemeroff, 2016). En este proyecto se pretende determinar de qué forma afecta una exposición crónica a ozono a un modelo animal de MI. Para ello se medirán parámetros que, como diversos estudios han demostrado, pueden modificarse por alguna de las dos condiciones.

Se ha observado que las crías que no han recibido cuidados adecuados presentan mayores niveles de corticosterona y ACTH tras la exposición a un estresor agudo (Kaufman et al., 2000). Debido a esto, es previsible una mayor concentración en sangre de estas hormonas tras la realización del test 'tanque de agua fría' en ratas que, no solo han sufrido PM, sino que también han sido expuestas a ozono. Además, se ha visto que sufrir PM aumenta el estado de ansiedad (Miller et al., 2016) por lo que, aparte de sufrir mayor aumento de hormonas tras la realización del test, estas ratas podrían presentar niveles basales de hormonas más altos que se correlacionasen con un aumento de la ansiedad en el laberinto en cruz.

Por último, podrían esperarse cambios epigenéticos en el grupo que será expuesto a PM. Por un lado, se espera un aumento de la metilación del exón promotor de GCr. Por otro lado, la metilación del promotor de AVP se prevé disminuida en los grupos que sufran PM y exposición a ozono.

En el presente trabajo se pretende clarificar de qué forma afecta la calidad del aire a personas que han sufrido MI, lo que podría suponer un dato a tener en cuenta a la hora de realizar un tratamiento adecuado.

\section{Bibliografía}

Andersen SL, Teicher MH. Delayed effects of early stress on hippocampal development. Neuropsychopharmacology: official publication of the American College of Neuropsychopharmacology. 2004; 29:1988-1993.

Buss C, Entringer S, Swanson JM, Wadhwa PD. The Role of Stress in Brain Development: The Gestational Environment's Long-Term Effects on the Brain. Cerebrum: The Dana forum on brain. Science. 2012: 4. 
Dobbing J, Sands J. Quantitative growth and development of human brain. Archives of disease in childhood. 1973; 48:757-767.

Europaress.es [Internet]. Internacional: Europapress; 2014 [actualizado el 02 jul. 2015; citado 27 feb. 2019]. Disponible en : https://www.europapress.es/internacional/ noticia-unicef-afirma-nino-muere-cada-cinco-minutos-mundo-causa-violencia-20141021062723.html.

Gilbert R, Widom CS, Browne K, Fergusson D, Webb E, Janson S. Burden and consequences of child maltreatment in high-income countries. Lancet. 2009; 373:68-81.

Hertzman C. Putting the concept of biological embedding in historical perspective. Proceedings of the National Academy of Sciences of the United States of America. 2012; 109 Suppl 2:17160-17167.

Kaufman J, Plotsky PM, Nemeroff CB, Charney DS. Effects of early adverse experiences on brain structure and function: clinical implications. Biological psychiatry. 2000; 48:778-790.

Kodavanti UP. Stretching the stress boundary: Linking air pollution health effects to a neurohormonal stress response. Biochimica et biophysica acta. 2016; 1860:2880-2890.

Lutz PE, Turecki G. DNA methylation and childhood maltreatment: from animal models to human studies. Neuroscience. 2014; 264:142-156.

Miller DB, Ghio AJ, Karoly ED, Bell LN, Snow SJ, Madden MC et al., Ozone Exposure Increases Circulating Stress Hormones and Lipid Metabolites in Humans. American journal of respiratory and critical care medicine. 2016; 193:1382-1391.

Miller DB, O'Callaghan JP. Neuroendocrine aspects of the response to stress. Metabolism: clinical and experimental. 2002; 51:5-10.

Nemeroff CB. Paradise Lost: The Neurobiological and Clinical Consequences of Child Abuse and Neglect. Neuron. 2016; 89:892-909.

Sapolsky RM. Stress and the brain: individual variability and the inverted-U. Nature neuroscience. 2015; 18:1344-1346.

Van der Kolk B. Commentary: The devastating effects of ignoring child maltreatment in psychiatry a commentary on Teicher and Samson 2016. Journal of child psychology and psychiatry, and allied disciplines. 2016; 57:267-270.

Who.int [internet]. Ginebra: Who; 2016 [actualizado 27 sep. 2016; citado 27 feb. 2019]. Disponible: https://www.who.int/es/news-room/detail/27-09-2016-who-releasescountry-estimates-on-air-pollution-exposure-and-health-impact.

Ediciones Universidad de Salamanca / @@ఠ $\quad$ FarmaJournal, vol. 5, núm. 1 (2020), pp. 65-73 